\title{
Ciudadanías movedizas: ciberpolítica y los dilemas de Twitter como esfera pública/privada ${ }^{1}$
}

\author{
Shifting Citizenships: Cyberpolitics and the Dilemmas \\ of Twitter as Public/Private Sphere
}

ÁLVARO ACEVEDO-MERLANO (Universidad de la Costa), ALICIA CHAMORRO MUÑOZ (Universidad Industrial de Santander - Universidad de la Costa) y MARGARITA QUINTERO-LEÓN (Universidad de la Costa)

Artículo recibido: 15 de octubre de 2020 Solicitud de revisión: 16 de mayo de 2021 Artículo aceptado: 16 de abril de 2021

Acevedo-Merlano, Álvaro, Chamorro Muñoz, Alicia y Quintero-León, Margarita (2021). Ciudadanías movedizas: ciberpolítica y los dilemas de Twitter como esfera pública/privada. Recerca. Revista de Pensament i Análisi, 26(2), pp. 1-23. doi: http://dx.doi.org/10.6035/recerca.5511

\section{Resumen}

El presente artículo propone una lectura de las ciudadanías digitales como movedizas desde una visión histórica y conceptual. Para realizarla, se desarrollan las siguientes partes: primero, una problematización sobre el concepto de ciudadanía digital desde una lectura crítica-histórica apostando por una comprensión compleja de esta que asume interacciones entre esferas públicas y privadas; segundo, tomando aspectos de la filosofía de la tecnología, analizamos la relación entre jóvenes y política a través de Twitter y, finalmente, una propuesta de la configuración de prejuicios y modelamientos que pueden darse en esta nueva esfera público-privada a partir de algunos aspectos vistos en la segunda vuelta de la campaña presidencial de Colombia en el año 2018. Una de las conclusiones a la que llegamos es que Twitter es una red sociotécnica que genera una forma movediza de conexión entre las esferas privadas y públicas, donde los jóvenes tienen agenciamiento y pueden generar procesos de resistencia a los modelamientos que las campañas políticas imponen.

Palabras clave: ciudadanía digital, Twitter, prejuicio y modelamiento, bots, esfera pública.

Los autores agradecen el apoyo financiero brindado por el Fondo Nacional de Financiamiento para la Ciencia, la Tecnología y la Innovación, Fondo Francisco José de Caldas, Minciencias y la Universidad de la Costa a través de la beca n.o 848 del 2019 y el proyecto n. 80740-095-2020. 


\begin{abstract}
This article proposes a reading of digital citizenships as these are shifting from a historical and conceptual perspective. We developed the following steps to carry it out: first, a problematization of the concept of digital citizenship from a critical-historical reading, betting on a complex understanding of it that assumes interactions between public and private spheres; second, taking into account aspects of the technology's philosophy, we analyze the relationship between young people and politics through Twitter, and finally, a proposal of prejudices configuration and modelling that can occur in this new public-private sphere based on some aspects seen in the second round of the 2018 Colombian presidential campaign. One of the conclusions we reached is that Twitter is a sociotechnical network that generates a shifting form of connection between the private and public spheres, where young people have assemblage and can generate resistance to the modelling imposed by political campaigns.
\end{abstract}

Key Words: digital citizenship, Twitter, prejudice and modelling, bots, public sphere.

\title{
INTRODUCCIÓN
}

Internet ha transformado las vidas humanas de tal forma que actualmente no nos es posible pensar en un tipo de interacción que no se haya visto permeada o, incluso, transformada por estas tecnologías caracterizadas por la disruptividad, la inmediatez y la ubicuidad, que crean redes en donde la realidad se concibe de una forma no lineal, asincrónica y colectiva. En el presente artículo nos ocupamos de cómo se han conceptualizado estas transformaciones en la esfera pública configurando unas nuevas formas de ser de la ciudadana mediadas por la tecnología, haciendo movediza la diferencia clásica entre lo que se consideraba terreno de lo privado y terreno de lo público.

En los contenidos de las redes sociales actuales, como Twitter, se evidencia una condición multimodal que incide en los hábitos de interacción de los usuarios mediante las hipermediaciones (Scolari, 2008). Así, la esfera público-privada que se genera en Twitter abarca nociones concernientes con el tiempo, el espacio, las relaciones sociales, las formas de obtener información y construir conocimiento. Asimismo, responde a la forma en que las personas se ven como individuos articulados a una red interconectada que cruza las fronteras de los llamados estados nacionales. Desde esta premisa partimos en el presente estudio para escoger Twitter como red de transformación de la ciudadanía digital.

En efecto, la personalización de las redes sociales, los filtros burbuja y la hipersegmentación (Pariser, 2011) juegan un papel fundamental en el proceso de 
la generación de tendencias políticas en las redes sociales y puede afectar de manera más profunda la configuración del joven como ciudadano digital, por una parte, a partir de un ingreso a las discusiones sobre lo público y político a edades menores y, por otra, siendo interceptados por las mismas campañas políticas, ya que estas hacen procesos de modelamiento de una imagen del joven que concuerda con sus líneas ideológicas. Este modelamiento marca la información y la construcción del mundo que cada sujeto recibe a partir de cada nueva notificación. Las afinidades se van tensionando y agravando en la esfera digital y es necesario un esfuerzo mayor para poder caer en la cuenta de los prejuicios que pueden estarse entretejiendo en cada actualización de las redes sociales.

Para analizar este fenómeno, primero conceptualizaremos y problematizaremos la forma en que se ha conceptualizado el ciudadano digital y, posteriormente, tomaremos como referencia las campañas políticas de los dos partidos de derecha e izquierda en Colombia que clasificaron a la segunda vuelta de las elecciones presidenciales del 2018, ya que acrecentó la polarización en la ciudadanía.

\section{CIUDADANÍA DIGITAL: PERPLEJIDADES Y DILEMAS}

En el análisis de Idhe sobre la filosofía de la tecnología (2004, 2009, 2010) podemos encontrar un punto fundamental para nuestro trabajo, a saber: la comprensión del mundo de la vida de la tecnificación. El mundo de la vida lo retoma Idhe desde la fenomenología y lo actualiza desde nuestras formas de habitar los mundos digitales. Así, para el autor, la comprensión del mundo de la vida, el espacio concreto del que emergen todas las experiencias humanas a las que remiten todas las preguntas de los hombres, ahora se ve configurado por las nuevas formas tecnológicas, que se imbrican interactuando y transformando las formas de comunicabilidad y toda nuestra comprensión clásica de la técnica y el uso de los artefactos.

Idhe (2004) sustenta que el artefacto permite una transparencia y, a la vez, una mediación en su uso. Como mediación, posibilita, a partir de su opacidad y transparencia, que sea usado para una tarea en específico. Por ejemplo, el bastón permite su uso en cuanto genera un rechazo o resistencia con el cuerpo y el suelo y, a la vez, una transparencia que lo hace parte del movimiento del cuerpo humano. También el martillo permite servir como herramienta en cuanto a la vez se hace parte de la mano y al mismo tiempo se asume como diferente y ejerce fuerza. 
Ahora bien, en el caso de las redes sociales nos encontramos ante otros procesos tecnológicos. La primera opción sería sostener que la red es una mediación entre sujetos que están interactuando y, por ende, es una plataforma que tendría la única función de conectarlos (como el bastón entre la mano y el piso). Pero parece que las redes son mucho más que esa herramienta que media y ejerce resistencia. Las redes se convierten en un medio tecnológico que no solo están mediando conversaciones naturales, sino que han generado su propia forma de comunicar y de configurar mundos (Idhe, 2010). Esto es cercano a lo que Ferraris (2008) afirma con respecto al teléfono móvil convertido en una mediación tecnológica que ha transformado las condiciones mismas de escribir, comunicarnos e interactuar con los otros, desde la misma pregunta que con anterioridad al teléfono móvil parecería estúpida y ahora, con estos dispositivos que cargamos a todas partes, nos parece esencial: ¿dónde estás? Sostenemos entonces que el desarrollo y el impacto de las redes sociales se conecta con las posibilidades de estos dispositivos cada vez más transparentes, más portables y determinantes como son el laptop y el teléfono móvil. Sherry Turkle muestra cómo internet transforma todas las formas clásicas de entender la técnica por parte de la filosofía y le da paso a un espacio de laboratorio de la identidad: «nosotros comenzamos a entendernos como fluidos, emergentes, descentralizados, múltiples e incluso un proceso» (Turkle, 2010: 280).

En este mismo orden de ideas, la generación de la tecnosfera público-privada, constituida por las redes sociales y soportada por los dispositivos cada vez más portables y robustos, determina las realidades en que nos movemos y ha transformado nuestras interacciones políticas, configurando lo que se conoce como la ciudadanía digital.

La ciudadanía digital abre un nuevo espacio para lo que denominamos tradicionalmente filosofía política. Lo anterior porque implica llevar al nivel de una nueva reflexión lo que hasta ahora se había considerado como sólido dentro del análisis sobre la ciudadanía, a saber: la conexión entre ciudadano y Estado; la configuración de un ciudadano como sujeto corpóreo y específico; las relaciones de poder que dentro de la ciudadanía se ejercen y, finalmente, las formas de comprensión de la isegoría y la isonomía (Arendt, 1997).

Ahora bien, a partir de lo anterior podemos establecer una serie de definiciones de ciudadanía y ciudadano digital teniendo en cuenta diferentes niveles: $a$ ) la tecnocrática, aquel sujeto que utiliza los medios tecnológicos para realizar actividades que anteriormente hacía de forma presencial y que están relacionadas con ejercer sus derechos ciudadanos de manera efectiva y eficiente; $b$ ) la participativa, la ciudadanía digital remite al sujeto político actual que configura 
una esfera pública a partir de la participación y transformación de los medios digitales, similar a lo que Dahlgren menciona como ciudadanía mediatizada $(2013)$; c) la ontológica, las ciudadanías digitales son nuevas formas de existencia que no pueden ser simplemente pensadas como una ampliación de las prácticas clásicas, sino son una verdadera transformación en lo que significa participar, manifestarse y construir la esfera pública (Chamorro y Palacio, 2014). De estas tres definiciones podemos decir que todas se dan en ámbitos sociopolíticos y están imbricadas en cualquier investigación contemporánea; por ende, lo mejor es tener una visión amplia del concepto de ciudadanía digital que permita asumir diferentes paradigmas y aceptar que parece ser un fenómeno tanto multifacético como dinámico.

\subsection{La dificultad de su historización}

Teniendo en cuenta esta última afirmación, es importante apuntar cómo se han dado diferentes lecturas sobre el fenómeno del ciudadano digital y su desarrollo histórico; así, podríamos hablar de cuatro grandes etapas de dichas investigaciones.

La primera etapa se da al inicio del siglo XXI y se caracteriza por una plétora de investigaciones que evidencian la sorpresa y la esperanza que marcaron la Primavera Árabe, la Revolución de Túnezy las manifestaciones tanto en España como en Estados Unidos. Para Zuckerman (2013), estos movimientos son impulsados por las necesidades y los problemas internos, como también por procesos de comunicación y relaciones internacionales que generan descontentos comunes y formas de interacción que llevarían a un cosmopolitismo digital. Como muy bien han mostrado diversos estudios, el proceso esperanzador de estos movimientos devino por las siguientes cualidades: 1) la condición pública de la configuración de las manifestaciones, sin que un grupo específico pudiera adjudicar ese poder ciudadano, 2) la creciente participación de grupos que tradicionalmente se habían considerado apolíticos, escépticos o excluidos y 3) la potencia explosiva de la repercusión en las manifestaciones transfronteras (Wolfsfeld, Segev y Sheafer, 2013; Smidi y Shain, 2017).

La segunda etapa, como un buen movimiento dialéctico, se caracteriza por las investigaciones que denotan un fuerte pesimismo. Estas lecturas interpretan $\mathrm{y}$ analizan las consecuencias dramáticas que los primeros movimientos mencionados tuvieron, mostrando sus debilidades. Por ejemplo, siguiendo a Mossberg, Toltbert y McNeal (2008), la falta de un grupo específico que se adjudique el 
movimiento complica los procesos de organización ciudadana, y las manifestaciones, aunque multitudinarias, terminan por ser cooptadas prontamente.

Sin duda, ambas etapas, que implicarán los ciberutopistas y los tecnopesimistas, son análisis precipitados, que responden específicamente a dar una explicación de lo que en ese momento estaba sucediendo; empero, los resultados de estas dos etapas desencadenan en una tercera etapa más determinada por el sentido tecnócrata de esta ciudadanía digital, es decir, de la forma en que la web como herramienta técnica media los procesos de ejercicio de la ciudadanía y su efectividad.

Finalmente, la cuarta etapa corresponde, dentro del amplio espectro de las ciencias humanas, al actual trabajo con relación a las estrategias de manipulación y la forma en que la verdad se configura dentro de los usos de las plataformas virtuales. Este tipo de trabajos surgen en torno a procesos políticos como el Brexit o las elecciones presidenciales de Estados Unidos (García y La Chicaíza, 2018). A esta etapa, lejos de importarle una versión moralista de la ciudadanía digital, le interesa el análisis de las interacciones de poder, que se dan en los ambientes virtuales y que impactan de manera directa las decisiones políticas (Chamorro y Palacio, 2014).

Es necesario destacar que estos fenómenos sociales siguen los tres rumbos de las TIC que plantea Pimienta (2007) sustentando su propuesta en escenarios indispensables para las nuevas interacciones de la sociedad: 1) la infraestructura, comprende la tecnología de los dispositivos capaces de transportar las señales, la información o los datos, el hardware, 2) la infoestructura, representa los software que contiene la lógica de los sistemas, contenidos y aplicaciones que operan sobre la infraestructura; 3) la infocultura, propia de la alfabetización digital del usuario, en este caso, del ciudadano que reúne las prácticas de los individuos bajo el entendimiento de las TIC para el desarrollo (TICpD), donde se apropia de la tecnología y la integra como un factor cultural de su cotidianidad (Quintero-León y Flores, 2020).

Por su parte, la actual ciudadanía digital asume la evolución de las TIC hacia las TAC; este tránsito permea las TEP, que son intrínsecas en los rumbos de configuración arriba mencionados. En este punto, las TIC dejan de ser herramientas con visión táctica para demostrar su nivel de influencia; se trata así de la perspectiva de un cibernauta autodidacta y curioso que le permite apropiarse del saber de forma personalizada gracias a la tecnología para el aprendizaje y el conocimiento TAC (Latorre, Castro y Potes, 2019). Por ende, la construcción colectiva de los significados que le aporta cada uno recrea verdades conjuntas y 
fomenta el descubrimiento de las funciones de las TIC. El ciberactivismo da resultados visibles en el pensamiento crítico (Ortiz, Quintero y Díaz, 2015) del cibernauta, que entiende las necesidades y las problemáticas internas, las cuales son divulgadas en una red de interconexión de posibles resultados mediante la tecnología que empodera e incita la participación (TEP) masiva del hoy ciudadano prosumidor (Islas, 2008) en las esferas de la política pública de un gobierno en línea.

\subsection{Hipermediatización de la esfera política a partir del modelamiento en Twitter}

La democracia representativa ha sido la solución dada para que las mayorías sean las que tomen las decisiones, pues la posibilidad de una democracia directa resulta complicada en términos operativos y, décadas atrás, la interconexión masiva como ocurre gracias a las TIC resultaba inimaginable. Sin embargo, teniendo presente la crisis de representatividad causada, entre otras cosas, por fenómenos como la corrupción, en la actual sociedad de la información las personas pueden acercarse un poco más a visibilizar su opinión a través de las redes y las interconexiones digitales (Guattari, 1996) que demandan la transparencia de los procesos y permiten mayor seguimiento, propio de una sociedad informada.

Así, los comentarios en imágenes, memes, videos y las interacciones como los me gusta, los comentarios y los retuits muestran las diversas maneras comunicativas que Twitter ha traído. En estas últimas, los sujetos se acercan a lo tecnológico, desembocando en un uso que logra la resignificación del sentido de la palestra política. Dicho panorama, como un gran reality show, permea las campañas políticas. Esto implica entender el factor de lo político como una estrategia de marketing, es decir, como un espectáculo posibilitado y facilitado por esa misma mediación tecnológica (Sánchez, 2005).

En este entorno postanálogo operan, al mismo tiempo, nuevas formas en las que se desarrolla el fenómeno político de manera espectacularizada, dando cabida a personajes como los showman, los mártires, los salvadores, los enemigos de la patria, los iconoclastas, los héroes, los terroristas o los carismáticos. Todo ese contexto de transmutación de la política pone en crisis la viabilidad de una democracia aumentada (Hidalgo, 2018) que logre depurar muchos de los inconvenientes que la democracia tradicional sigue arrastrando a los entornos digitales. 
En las redes sociales se expone la intimidad como un espectáculo (Sibilia, 2008), elaborando narrativas en las que las personas tienden a circunscribir sus experiencias y su contexto inmediato, mientras construyen el imaginario de su realidad sociopolítica a través de las pantallas. Mediante estos ejercicios, los sujetos se desligan de la realidad concreta que los interpela, pues dichas estrategias de marketing político pueden generar un impacto mayor a través de una ecología mediática que lo permita, en tanto esa realidad se transforme, edite, construya y reconstruya, desde una mediación tecnológica.

En estas relaciones es evidente la superación de la antigua distinción moderna entre sujeto y objeto, pues dichas categorías divisorias no encajan en las reciprocidades de las mediaciones en la red, que resultan ser más bien rizomáticas y efectuadas por agentes, indistintamente de si son o no humanos, que cumplen su objetivo de proporcionar un ambiente verosímil con quien interactúa desde el tuit. Teniendo en cuenta lo anterior, se puede entender Twitter como una red sociotécnica (Latour, 2008), que propicia actualmente las relaciones entre humanos y no-humanos, en donde los bots pueden ser considerados actantes con capacidad de agenciamiento, esto es, generadores emocionales que logran incidir directamente en la realidad de los actores humanos, a través de los tuits con los que son programados. Cabe aclarar que, cuando se hace referencia a un bot, este no es más que un perfil de usuario con un software integrado que automatiza las interacciones con otros usuarios. En suma, utilizando bots, ${ }^{2}$ el usuario que lo desee puede continuar eficazmente con el proceso de tuitear a destiempo, lo que le permitirá seguir existiendo en la virtualidad, posteando tuits preprogramados o repitiendo tuits publicados previamente. Todo esto con la única intención de seguir el hilo conductor discursivo de la idea que se pretende mantener en debate y consolidar la presencia en el ciberespacio.

Veamos detenidamente este punto. Cuando analizamos la dinámica en la que se da una discusión o un debate tradicional, cara a cara, en el mundo análogo, se puede observar que, durante el tiempo en el que ocurre, se confrontan posturas en las que cada uno de los participantes de la discusión desea convencer al otro, persuadiéndolo para que ceda a su idea. Sin embargo, puede llegar un momento en el que, en medio del cruce de palabras, no se aporte concretamente nada novedoso a las ideas planteadas inicialmente por los sujetos, sino que solo

Para conocer más sobre la naturaleza y las características de los trols y los bots, véase: https://ijnet.org/es/story/todo-lo-que-hay-que-saber-sobre-trolls-bots-y-botnets y https://medium.com/dfrlab/botspot-twelve-ways-to-spot-a-bot-aedc7dgc110c 
se continúe acudiendo, en algunos periodos, a recursos retóricos cíclicos, utilizados con el propósito de mantener el hilo discursivo en la conversación. En la ausencia de argumentaciones válidas y en la presencia de oraciones extensas cuasi automáticas, ambos mitigan la conversación, mientras se les ocurre algo realmente contundente que esté en oposición a los planteamientos de su interlocutor, para así tumbar su postulado. Lo descrito es bastante similar a lo que sucede en la interacción en Twitter cuando se hace uso de un bot. Lo que diferencia una forma de la otra es que en Twitter ocurre de manera asincrónica y en ausencia de alguno o de ambos interlocutores.

De ese modo, mientras a las personas inmersas en ese proceso se les ocurre un gran tuit, dejan a los bots en línea para que continúen visibilizando las ideas características del discurso que les interesa mantener. Estos perfiles podrían concebirse como un sujeto simulado, que se usa como una estrategia que permite mantener al otro conectado y generar la percepción de participación activa. Como resultado, el bot cumple la función de enganchar a un público que siempre debe estar preparado y predispuesto a unas potenciales interacciones, que oscilan entre las ausencias, las presencias, las simulaciones y las representaciones de unos agentes que participan en la construcción del contexto en el que se desarrollan los debates. Más aún, los bots pueden ser usados de manera fraudulenta

por parte de los gobiernos y los partidos políticos para manipular la opinión pública e interferir en la comunicación política para solicitar contribuciones o votos, fingir una mayor popularidad en una red social, perturbar la estrategia de comunicación de los rivales o lanzar falsas opiniones para influir en el electorado (Calvo, 2020: 137).

Igualmente, la práctica de falsos seguidores es cada vez más común en las estrategias políticas (Serrano, 2016; García-Guerrero, 2019), lo que desvanece uno de los atributos más fuertes de la sociedad de la información: la transparencia. El 2016 fue escenario de esto en las elecciones presidenciales entre Clinton y Trump (Semana, 2018). En el continente africano, las elecciones de Tanzania en el 2015 fueron objeto de disputas políticas en el escenario físico y virtual: en promedio, el 50,7\% de los seguidores en Twitter de los candidatos vicepresidenciales eran falsos y las tendencias en redes sociales fueron infladas (Shayo y Kersting, 2016). En Latinoamérica, en las elecciones del 2014 en Brasil, se encontraron más de cien perfiles falsos creados desde el 2012 por la agencia Facemedia, costeada por el Partido de la Social Democracia Brasileña (Gragnani, 
2017). El caso colombiano no es la excepción con respecto a malas prácticas digitales, tanto en el sector público como privado. Las agencias de seguridad y marca Adalid y LOOR, en el 2018, calificaron de sospechosos a varios seguidores de los entonces candidatos presidenciales, evidenciando que el $19 \%$ eran falsos perfiles (Twitter Audit, 2021). En el caso de ambos candidatos las cifras no son menores, ya que en Colombia la guerra sucia en redes sociales surge del uso para ventaja propia de los algoritmos de las redes sociales para ampliar el impacto de los mensajes y la creación de tendencias políticas (Carreazo, 2020).

Lo anterior nos deja ante la perplejidad sobre cómo entender estas ciudadanías digitales tan movedizas como complejas.

\section{JÓVENES Y POLÍTICA: INTERACCIONES ENTRE ESFERAS}

Es clásica la aportación de Castells (2009) sobre un tipo de activismo digital que el autor ha relacionado con un movimiento general anticapitalista en el que la conexión de red ha servido en el ámbito estratégico, organizativo y normativo. A principios de la década de los noventa ya hacía un temprano diagnóstico, en el sentido de que «actores sociales y ciudadanos de todo el mundo están usando ya esta nueva capacidad de las redes de comunicación para hacer avanzar sus proyectos, defender sus intereses y reafirmar sus valores» (Castells, 2009: 91).

En este caso, el ciudadano, entendido como aquel sujeto con espacio para la palabra y la configuración del mundo común, cuenta ahora con diferentes plataformas de internet como nuevos espacios públicos de ideas. Así, retomando, la ciudadanía digital implica procesos de participación por medios digitales y usos de la red para expandir los actos que antes se llevaban a cabo de manera presencial, generando una sociedad en línea donde es posible desarrollar y participar en la esfera pública en orden de cumplir sus responsabilidades laborales y económicas, como también para abrir sus posibilidades sociales y constituir un mundo de la vida desde la tecnificación (Mossberg, Toltbert y McNeal, 2008).

El privilegio de las condiciones de horizontalidad de las relaciones ha sido uno de los grandes baluartes por los cuales se consideró que las nuevas tecnologías ofrecían un campo de cultivo favorecedor a las democracias; empero, parece que dentro de estos marcos de horizontalidad se esconden formas verticales de 
manejo de la información, formas persuasivas y de manipulación igual de fuertes que las existentes en los procesos de la democracia tradicional que afectan la inclusión de los jóvenes en la política.

\subsection{La relación entre fronteras y territorio con el ciudadano joven digital}

Tras la masificación de internet como herramienta de información y posteriormente de comunicación, nace el e-government aludiendo a las TIC como canal de comunicación directa con sus públicos objetivos, los ciudadanos del mundo y las empresas, para atender sus necesidades con servicios online (Alfaro, Bustos, González y Loroño, 2005). Este concepto tiene como finalidad la e-democracy, fase de alta participación ciudadana capaz de inferir en la toma de decisiones de un gobierno en línea (Pozo, 2013).

En respuesta a la tendencia de un e-government o gobierno electrónico, las Naciones Unidas, por medio de su Departamento de Asuntos Económicos y Sociales (UN DESA), viene generando desde el 2001 un escalafón bianual con los 193 países que la integran, en busca de establecer cuáles son los países abanderados de la gestión transparente, la prestación de servicios online y la rendición de cuentas. El escalafón inició en el 2003 con Estados Unidos en el top 1 y, 17 años después, el líder del mundo en e-democracy es Dinamarca.

El ámbito de la política se está transmutando en un proceso de imaginarios colectivos que visibilizan problemas de otras nacionalidades que afectan las formas de vida de varios países; así, dichos imaginarios comienzan a generar procesos de ciberactivismo que dislocan la idea de ciudadanía, determinada por las fronteras del Estado que se convierten en movimientos que aprenden unos de los otros. ${ }^{3}$ Un fenómeno que es más claro en los jóvenes y su ingreso a la política, donde parece que la apatía tradicionalmente asignada a este grupo se difumina a partir de sus interacciones en el espacio virtual. Sin embargo:

La falta de participación cívica no debe considerarse como una simple cuestión de apatía ciudadana, sino que debe entenderse dentro del contexto de los dilemas más comunes en la democracia de la modernidad avanzada (Dahlgren, 2013: 48).

Un ejemplo del poder de trascender las fronteras de las preocupaciones de la esfera pública a partir de una constitución del cosmopolitismo es el hashtag \#bringbackourgirls, campaña desarrollada en el 2014 como una forma de hacer consciente al mundo de los dramas vividos en Nigeria causados por los grupos extremistas de Boko Haram (Penney, 2017: 5-10). 
Parece que los jóvenes encuentran su forma de conectarse con lo político desde las discusiones en redes. Ya no se puede desconocer la importancia y el impacto que tienen Facebook, YouTube y Twitter (Curran, 2005) dentro de la esfera pública juvenil. Esto nos lleva a preguntarnos si es Twitter un espacio de la configuración de la esfera pública donde ingresan los jóvenes como nuevas ciudadanías. Como Habermas ha afirmado en los últimos trabajos, la esfera pública es un espacio tanto de lo común como de la lucha de poderes, un espacio multiforme donde diferentes ideologías se pueden hacer presentes y se requiere un deseo mutuo de comprensión para poder construir los aspectos políticos de intereses comunes, en relación y teniendo en cuenta las diferencias de los grupos sociales que están presionando para ser escuchadas y valoradas (Habermas, 1998). Parece ser que los jóvenes crean un poder dentro de este juego de luchas y son también espacios de disputa para las formas políticas ya constituidas tradicionalmente.

Ahora bien, la esfera solitaria propuesta por Dahlgren (2000, 2013) y Papacharissi (2010) puede ser de interés para entender este fenómeno. Una esfera que pertenece a un interés político, pero con cierto escepticismo de la generación de comunidad política o del efectivo desarrollo público de la ciudadanía. El fenómeno al que nos enfrentamos es la existencia de un enterarse desde casa de lo que sucede y una configuración de un yo político aislado que asume las discusiones y debates sin salir de casa, ni discutir con otros ciudadanos. Es importante recalcar que la esfera privada no implica un aislamiento en el sentido clásico en el que se diferenciaba lo privado de lo público, sino que, con la emergencia de las redes sociales, parece que se conecta este sujeto en su privacidad con las discusiones políticas, sociales y económicas en una extraña conexión entre el espacio privado del individuo y el espacio público, que podría llevar a un colapso entre los límites de las esferas.

Cabe apuntar que, si bien parecen ser estas esferas mezcladas, por decirlo de alguna manera, a lo que los jóvenes entran en sus primeras participaciones políticas, existe una dificultad profunda que separa las redes sociales de la esfera pública tradicional. La dificultad de entender las redes sociales como meramente sociales es lo que no deja a la vista las estrategias de manipulación que dentro de ellas se encuentran, los dominios de poder y la visibilidad que se maneja (Rincón, 2011). Parece que hablar de una red social sea simplemente una conexión entre la isonomía de individuos, pero toda red social está al mismo tiempo determinada por monopolios económicos y formas de poder que no ce- 
den en las formas hegemónicas de repartir y dar visibilidad, volviendo a la perspectiva de Idhe de la herramienta técnica: por más transparente que parezca, Twitter es una herramienta manejada y usada.

\subsection{Jóvenes universitarios y ciudadanías digitales}

En este contexto, podemos preguntarnos por la relación entre Twitter y la población universitaria: ¿pueden ser las redes sociales un espacio que permita el enganche político de los jóvenes? Existe una relación entre el deseo de ser más participativos y lo que se cuela en cada una de sus interacciones en las redes y también parece ser que las redes permiten una apropiación mayor de conocimiento (Krueger, 2002; Bimber, 2003; Tolbert y McNeal, 2003).

Por otro lado, pese a las brechas digitales (Pimienta, 2007) que vive un país del tercer mundo, como Colombia, y las dificultades que un gran porcentaje se encuentra para acceder a la información, la población universitaria juvenil parece ser un grupo homogéneo en su relación con internet. La mayoría, en condiciones normales, tiene acceso a internet ya sea propio o por medio de las redes universitarias y cuenta con acceso a dispositivos móviles o computadores en el marco normal de su día a día a partir de las ventajas que ofrece el campus universitario.

Las formas de comunicación en internet, como los chats, las noticias en línea e incluso el correo electrónico, se convierten en un nuevo espacio de la palestra pública en el cual las personas no solamente exponen sus puntos de vista y se informan, sino que también deciden su voto. Además, existen las versiones falsas, comunes en la población juvenil, de la actividad cívica, donde el ciudadano cree que con el mero compartir o dar un me gusta o replicar está ejerciendo de manera directa su ciudadanía, cuando realmente el impacto puede ser nulo. Cabe preguntarse hasta qué punto dar me gusta, retuitear, compartir un enlace y publicar una foto o un meme realmente contribuye o genera un verdadero debate que ha mostrado un desequilibrio contundente entre la votación real y el apoyo de campañas en redes sociales. La forma en la que los jóvenes se relacionan e interactúan a través de Twitter —en donde la inmediatez es un elemento fundamental - logra modificar la concepción que se tiene de la participación, la política y la ciudadanía, además de otros elementos que configuran la vida cotidiana. En ese sentido, Meneses, Ortega y Urbina (2014: 86) exponen que: 
las plataformas digitales permiten a los jóvenes ciudadanos un compromiso líquido, aludiendo a Bauman (2010): observo, no me comprometo y me desafano con un clic. No obstante, la utilización electoral de tales medios refleja solo una cara particular de su alcance vinculatorio con la participación política.

Por el contrario, Viché (2015) analiza el fenómeno desde la acción y plantea la ciberanimación como una praxis sociocultural que se masifica entre los jóvenes, quienes desde la

comunicación interactiva, organización social, empoderamiento colectivo y creación de representaciones identitarias solidarias, se nos presenta como una práctica estratégica de conectividad, cooperación, análisis crítico, generación de identidades y cambio social (Viché, 2015: 27).

Se busca un pensamiento colectivo que contribuya a una mejor realidad del colectivo ciudadano, partiendo de una estructura organizada y continua de personas comprometidas que dinamiza las conversaciones en el ciberespacio en pro de los objetivos que las unen.

Así las cosas, aún no es clara la influencia real de los procesos participativos de las redes en el voto actual de la población juvenil. Cuando un joven se encuentra frente a su computadora, solo en su habitación, utilizando los periféricos de entrada para acceder al mundo virtual donde se da el debate en el que desea participar, se genera una simulación de contacto que produce nuevas formas de sociabilidad residual mediatizada y, a la vez, influenciada por el mundo análogo, difuminando las fronteras que separan la esfera pública de la privada. Lo anterior muestra cómo «la línea divisoria creada por la pantalla entre el mundo virtual y el análogo se desdibuja, pues ambos repercuten entre sí» (Acevedo-Merlano y Maya, 2016: 135). Se ofrece así la posibilidad de que las personas interactúen al mismo tiempo que están solas, produciendo subjetividades como el Homo Twitter,

cuya existencia es individual, tan individual como lo específicamente humano, como la existencia auténtica cuyos componentes esenciales - la diferencia y la libertad - solo se realizan en el espacio público. Frente a la tendencia de las democracias liberales occidentales a reducir el discurso público a pura mediación de intereses igualmente particularistas, frente a la precariedad de una integración social y étnica cada vez más débil y frente a la tendencia de los sistemas políticos democráticos a legitimarse en los términos, siempre aleatorios, de la prosperidad económica, la integración de las sociedades complejas ya no puede fundarse sobre los principios democráticos, tal y como los conocemos (Cansino, 2017: 400-401). 
A partir de lo visto anteriormente, dos aspectos quedan claros: la esfera privada y la esfera pública se entrelazan cada vez al perder su especificidad; la población joven encuentra su primera ágora en los espacios de la virtualidad determinando un ingreso a las discusiones políticas antes incluso de tener acceso al voto. Segundo, estas condiciones no son ajenas a las campañas políticas que tratan de construir una imagen del joven a partir de la cual aspiran impactar a tal población. Esta constitución de la imagen del joven no se desliga de las mismas ideas que constituyen la ideología de los partidos.

Para analizar este fenómeno tomamos como referencia las campañas políticas de los dos partidos, de derecha e izquierda, en Colombia que clasificaron a la segunda vuelta de las elecciones presidenciales del 2018, donde se vieron enfrentados Gustavo Petro por el partido Colombia Humana e Iván Duque por el partido Centro Democrático. Esto representó en el país la disputa por el poder entre la izquierda y la derecha, acrecentando la polarización en la ciudadanía.

Esta división no fue ajena a las discusiones que se presentaron desde Twitter, donde gran parte de los usuarios, antes de centrarse en comentarios que generaran un debate limpio, arremetieron con insultos o memes a quienes no compartían su posición. Incluso las fake news fueron material para defender sus opiniones. Por otra parte, desde los perfiles de los candidatos y sus respectivos partidos políticos se construyeron discursos dirigidos a captar la atención de los jóvenes votantes mediante propuestas que fueran de su interés como: primer empleo, medio ambiente, paz, transparencia, género y educación. Así, estos actores tuvieron mayor interacción desde cada una de las publicaciones que más los identificaba y que en muchos casos era la continuación de lo que otros habían presenciado desde las plazas públicas, pues mediante fotos y videos se mostraba a la juventud siendo participe de los eventos y las actividades políticas llevadas a cabo desde el mundo análogo (Acevedo-Merlano, Chamorro y Quintero-León, 2020).

Durante esta contienda por el poder, la ideología de los partidos desde Twitter y en relación con la juventud mostró dos perspectivas: por Colombia Humana se vio un lenguaje más inclusivo, que llamaba al liderazgo y a una educación superior de calidad que, como parte de su estrategia, sumó a los académicos que apoyaron la campaña de Petro; mientras que por el Centro Democrático los trinos fueron dirigidos a impulsar y apoyar el acceso a una educación técnica o tecnológica que buscó influir en la opinión de los jóvenes con el acercamiento a figuras públicas del entretenimiento y del deporte que acompañaron a Duque en su candidatura. Ambas posturas se convirtieron en centro 
de discusiones que, aisladas del contenido publicado o unidas a una conversación específica, mostraron otra forma de participación, que los hace pertenecer a la discusión más trinada del momento y que demuestra una variación entre el ágora pública y la digital (Acevedo-Merlano, Chamorro y Quintero-León, 2020).

\section{CONCLUSIONES: PREJUICIOS Y MODELAMIENTO}

Los actuales hábitos juveniles generados por la integración tecnológica en esta esfera público-privada superan el contexto virtual y lo extrapolan al entorno análogo de la cotidianidad, el cual sufre modificaciones por la tecnología reticular. Esta última, al ser adaptada, incide en las prácticas socioculturales y en las relaciones entre individuos, cambiando costumbres y creando hábitos establecidos, en disrupciones y procesos simultáneos, intemporales y fraccionarios. Así, en esta nueva realidad, lo invariable en la constitución de costumbres no es la reiteración o la continuidad duradera de actividades o acciones; por el contrario, es la ruptura de las prácticas, la transformación y la adaptabilidad.

Twitter puede incidir en las maneras en que se elaboran los enunciados desde modalidades discursivas públicas y privadas, gracias a la facultad de anonimización que le otorga al discurso oculto (Scott, 2004). En ese sentido, en la esfera público-privada las vivencias mediatizadas y las formas de responder cambian y se conjugan, creando nuevos modos de interrelación que devienen de la virtualización de una micropolítica (Deleuze y Guattari, 2002).

Cabe destacar que las acciones políticas a través de Twitter son motivadas e impulsadas por emociones como la frustración o la ira. En el caso del activismo político en red, estos son los principales incentivos para que los jóvenes decidan acercarse y participar en la apropiación y la integración del nuevo escenario tecnológico, el cual resignifica las maneras de relacionamiento basadas en un mismo objetivo dentro de la esfera público-privada (Gravante, 2012; Sabater, 2014; Clua, Ferran-Ferrer y Terren, 2018).

No obstante, el principal propósito de muchas declaraciones realizadas en Twitter es manipular las emociones de los usuarios, con el único objetivo de generar molestias y discusiones violentas sobre temáticas sensibles o polémicas. De esta forma, a través de la provocación, se logra una respuesta emocional, destruyendo así la posibilidad de un debate real. Twitter forja mutaciones comunicativas en lo cotidiano, las cuales, al ser advertidas y adoptadas, logran reconfigurar el significado de las interacciones gestadas dentro y fuera de internet, 
desde procesos descentralizados, mediatizados, remotos y rizomáticos, que niegan la dinámica de un tiempo lineal heredero de la episteme moderna, sustentada sobre concepciones secuenciales, binarias, racionales y totalizantes, pero hoy desafiadas en una esfera que combina de manera movediza lo público y lo privado.

A partir del trabajo desarrollado, la gran pregunta que queda es: ¿cuál es el nivel de reflexión y agenciamiento que tienen los jóvenes dentro de sus procesos de participación en las nuevas palestras públicas determinadas por la tecnología? Por una parte, sería absurdo intentar pensar en una comprensión de esa reflexión y agenciamiento desde una perspectiva de la autonomía absoluta sustentada en lo que sería ese sujeto moderno. Antes bien, asumiendo un mundo interconectado, toda reflexión implica un proceso de darnos cuenta de la interconexión y la necesidad de reconocimiento de diferentes alteridades. Por otra parte, el agenciamiento no se puede entender por fuera de una serie de relaciones de poder que se van marcando a partir de unos prejuicios que son utilizados claramente por las campañas políticas para provocar mayor recordación y posicionamiento.

Podemos sostener que estos procesos se alimentan de los prejuicios identitarios (Fricker, 2017). Como tal, el problema del prejuicio identitario radica en que posibilita la exclusión de aquellos grupos que no pertenecen identitariamente y hace que cada vez se cierre más el horizonte de comprensión del otro (Medina, 2013). El joven puede exponerse a ser moldeado tal cual los valores de un partido político con fuertes tradiciones y la campaña utiliza ciertas estrategias políticas para mantener los prejuicios que le favorecen. El problema radica en que, cuando el prejuicio cala, las comunidades comienzan a desoír a los otros, negándose la posibilidad de interpelación y, en casos graves, generando injusticias epistémicas (Fricker, 2017). Empero, los jóvenes tienen un nivel de agenciamiento y pueden generar procesos de resistencia a tales modelamientos que tanto la derecha como la izquierda quieren hacer de ellos. Estos procesos de agenciamiento vienen desde las respuestas a los tuits hasta los usos de los mismos hashtags en formas divergentes y subversivas a lo que era deseado por la campaña.

Además del contenido, que es el foco de la discusión, los jóvenes también deben tener mucho cuidado con las fuentes y la confiabilidad. Si logran encontrar otras fuentes, es necesario hacer que estas correspondan a diversas tendencias ideológicas para forjar un criterio propio; sería lo que Medina ha denominado la necesidad de fricciones epistémicas como la apertura a la pluralidad de voces que se pueden agenciar dentro de las redes sociales, convirtiendo 
el espacio ciberpolítico en un ámbito que permita la pluralidad y el desarrollo de virtudes epistémicas tales como la lucidez, la responsabilidad activa y la apertura de mente (Medina, 2013). Ante esto, quedan las preguntas: ¿cuál es el espacio que el nuevo modelo democrático tiene para la participación de las juventudes? y ¡cómo los jóvenes reclamarán su soberanía, se convertirán las juventudes en los principales veedores de la gestión pública mediante el ciberactivismo o la ciberanimación?

\section{BIBLIOGRAFÍA}

Acevedo-Merlano, Álvaro y Maya, Natalia (2016). Difusión de realidades: comunidades virtuales presentes en los videojuegos de rol en línea. (Caso Aguabrial-Dofus, Periodo 2012-2013). Education in the Knowledge Society, 17(2), 133-148.

Acevedo-Merlano, Álvaro, Chamorro, Alicia y Quintero-León, Margarita (2020). La imagen del joven en Twitter como objetivo de persuasión en campañas presidenciales. En Mancinas-Chávez, Rosalba (Ed.). Universidad, innovación e investigación ante el horizonte 2030 (588-589). Sevilla: Egregius.

Alfaro, Rodrigo, Bustos, Guillermo, González, Alejandra y Loroño, Joseba (2005). El E-goverment. En Introducción al gobierno electrónico: actores y dimensiones (13-22). Valparaíso: Ediciones Universitarias Valparaíso.

Arendt, Hannah (1997). Qué es la política. Barcelona: Paidós.

Bimber, Bruce (2003). Information and American Democracy. Technology in the Evolution of Political Power. Cambridge: Cambridge University Press.

Calvo, Patrici (2020). Democracia aumentada: un ecosistema cibernético para una participación política basada en algoritmos. Ápeiron: estudios de filosofía, 12, 129-141.

Cansino, César (2017). Viejas y nuevas tesis sobre el Homo Twitter. Revista Mexicana de Ciencias Políticas y Sociales, LXII, 231, 389-405. 
Carreazo, Diana (2020). Anatomía política de Twitter en Colombia: Elecciones presidenciales 2018. Tesis maestría. Bogotá: Universidad Nacional.

Castells, Manuel (2009). Comunicación y poder. Madrid: Alianza.

Chamorro, Alicia y Palacio, Manuel (2014). Ciudadanía digital: inclusión y exclusión. Análisis de la habitabilidad de los MOOC. Revista Análisis, 46(85), 303-324.

Clua, Anna, Ferran-Ferrer, Núria y Terren, Ludovic (2018). El impacto de los jóvenes en la esfera pública: La disolución del Consejo de la Juventud de España en la prensa y en Twitter. Comunicar, 26(55), 49-57.

Curran, James (2005). Rethinking the media as public sphere. En Dahlgren, Peter y Sparks, Colin. Communication and citizenship. Journalism and the public sphere (27-56). New York: Routledge.

Dahlgren, Peter (2000). Television and the Sphere Public. Citizenship, Democracy and the Media. London: SAGE Publications.

Dahlgren, Peter (2013). The Political Web. Media, Participation and Alternative Democracy. New York: Palgrave McMillan.

Deleuze, Gilles y Guattari, Félix (2002). Mil Mesetas: Capitalismo y esquizofrenia. Valencia: Pre-Textos.

Ferraris, Mauricio (2008). ¿Dónde estás? Ontología del móvil. Barcelona: Marbot.

Fricker, Miranda (2017). Injusticia epistémica. Barcelona: Herder.

García, Mario y Chicaíza, Liliana (2018). Brexit, Paz y Trump: enseñanzas para los economistas. Revista de Economía Institucional, 20(38), 129-156. Recuperado de: https://ssrn.com/abstract $=3157254$.

García-Guerrero, Jorge (2019). Redes sociales e interés político: frecuencia con la que se comparte información sin confirmar en Quito. $\mathrm{ICONO}_{14}, 17(2), 231-253$.

Gragnani, Juliana (2017). Exclusivo BBC: la investigación que revela el ejército de perfiles falsos usados para influir en las elecciones de Brasil. BBC Brasil. Recuperado de: https://www.bbc.com/mundo/ 
noticias-america-latina-42270499 [Consultado el 11 de octubre de 2020].

Gravante, Tommaso (2012). Ciberactivismo y apropiación social. Un estudio de caso: la insurgencia popular de Oaxaca. Sociedade e Cultura, 15(1), 51-60.

Guattari, Félix (1996). Caosmosis. Hacia un nuevo paradigma estético. Buenos Aires: Manantial.

Habermas, Jurgen (1998). Facticidad y validez. Madrid: Trotta.

Hidalgo, César (2018). Una idea osada para reemplazar a los políticos.

Nueva York: TED. Recuperado de: https://www.ted.com/talks/ces ar_hidalgo_una_idea_osada_para_reemplazar_a_los_politicos

[Consultado el 15 de marzo de 2020].

Ihde, Don (2004). Los cuerpos en la tecnología. nuevas tecnologías: nuevas ideas acerca de nuestro cuerpo. Ciudad de México: UOC.

Ihde, Don (2009). Postphenomenology and Technoscience. New York: Suny Press.

Ihde, Don (2010). Heidegger's technologies. Postphenomenological perspectives. New York: Fordham University Press.

Islas, José (2008). El prosumidor. El acto comunicativo de la sociedad de la ubicuidad. Palabra clave, 11(1), 29-39.

Krueger, Brian (2002). Assessing the Potential of Internet Political Participation in the United States Resource Approach. American Politics Research, 30(5), 476-498.

Latorre, Edimer, Castro, Katherine y Potes, Iván (2019). De los medios a las mediaciones en la educación. En Las TIC, las TAC y las TEP: innovación educativa en la era conceptual (37-46). Bogotá: DGP.

Latour, Bruno (2008). Reensamblar lo social. Una introducción a la teoría del actor-red. Buenos Aires: Manantial.

Medina, José (2013). The Epistemology of Resistance. Gender and Racial Oppression, epistemic injustice, and resistant imaginations. New York: Oxford University Press.

Meneses, María, Ortega, Enedina y Urbina, Gustavo (2014). Jóvenes conectados y participación político ciudadana en el proceso 
electoral de México en 2012. Versión. Estudios de Comunicación y Política, 34, 71-92.

Mossberg, Karen, Toltbert, Caroline y McNeal, Ramona (2008). Digital Citizenship. The Internet, Society and Participation. Massachusetts: The MIT Press.

Ortiz, Gloria, Quintero, Sandra y Díaz, Ángela (2015). Las TIC-TACTEP: un referente para la educación policial. Revista Logos, Ciencia \& Tecnología, 6(2), 241-245.

Papacharissi, Zizi (2010). A Private Sphere. Democracy in a Digital Age. Cambridge: Polity Press.

Pariser, Eli (2011). The Filter Bubble: What the Internet is hiding from You. New York: The Penguin Press.

Penney, Joel (2017). The Citizen Marketer. Promoting Political Opinion in the Social Media Age. New York: Oxford University Press.

Pimienta, Daniel (2007). Brecha digital, brecha social, brecha paradigmática.

Recuperado de: http://funredes.org/mistica/castellano/ciberoteca/tematica/br echa_paradigmatica.pdf.

Pozo, Kelly (2013). Retos digitales: A propósito de gobierno en línea en el Atlántico. En Flores, Pamela (Ed.). Atlántico digital: logros y desafíos: procesos para una ciudadanía digital (55-78). Barranquilla: Editorial Universidad del Norte.

Quintero-León, Margarita y Flores, Pamela (2020). Evolución del eGovernment en Colombia: el caso del Departamento del Atlántico. Informação \& Sociedade: Estudos, 30(4), 1-14.

Rincón, Omar (2011). Mucho ciberactivismo... pocos votos: Antanas Mockus y el Partido Verde colombiano. Nueva sociedad, 235, 74-89. Sabater, Carmen (2014). La vida privada en la sociedad digital. La exposición pública de los jóvenes en internet. Aposta. Revista de Ciencias Sociales, 61, 1-32.

Sánchez, Luís (2005). El marketing político y sus consecuencias para la democracia. Comunicación y Sociedad, 4, 11-38. 
Scolari, Carlos (2008). Hipermediaciones: elementos para una teoría de la comunicación digital interactiva. Barcelona: Gedisa.

Scott, James (2004). Los dominados y el arte de la resistencia. Discursos ocultos. México: Era.

Semana (2018). Investigadora de falsos seguidores en campañas presidenciales revela su visión. Semana. Recuperado de: https://www.semana.com/edicion-impresa/informeespecial/articulo/radiografia-de-seguidores/255494/[Consultado el 12 de octubre de 2020].

Serrano, Pascual (2016). La batalla de la izquierda y las redes sociales. Educare, 20(66), 367-373.

Shayo, Deodatus y Kersting, Norbert (2016). An examination of online electoral campaigning in Tanzania. En 2016 Conference for EDemocracy and Open Government (CeDEM) (69-76). doi: 10.1109/CeDEM.2016.19.

Sibilia, Paula (2008). La intimidad como espectáculo. México: Fondo de Cultura Económica.

Smidi, Adam y Shahin, Saif (2017). Social Media and Social Mobilization in the Middle East: A Survey of Research on the Arab Spring. India Quarterly, 73(2), 196-209. doi: 10.2307/48505308.

Tolbert, Caroline y McNeal, Ramona (2003). Unraveling the effects of the Internet on political participation? Political Research Quarterly, $56(2), 175-185$.

Turkle, Sherry (2010). Alone Together. Why we expect more from technology and less from each other. New York: Basic Books.

Twitter Audit (2021). How many of your followers are real? Twitter Audit. Recuperado de: https://www.twitteraudit.com/ [Consultado el 31 de marzo de 2021]

Viché, Mario (2015). El empoderamiento de los ciudadanos en internet. Revista Internacional de Pensamiento Político, 10, 85-100. Recuperado de: https://www.upo.es/revistas/index.php/ripp/article/view/3587. 
Wolfsfeld, Gadi, Segev, Elad y Tamir, Sheafer (2013). Social Media and the Arab Spring: Politics Comes First. The International Journal of Press/Politics, $18(2), 115-137$.

Zuckerman, Ethan (2013). Rewire: Digital Cosmopolitans in the Age of Connection. New York: W. W. Norton. 